\title{
Attractor Density Models with Application to Analyzing the Stability of Biological Neural Networks
}

\author{
Christian Storm and Walter J. Freeman \\ University of California at Berkeley, Graduate Group in Biophysics, \\ 9 Wellman Court, Berkeley, CA USA 94720 \\ raystorm@uclink4.berkeley.edu, wfreeman@socrates.berkeley.edu \\ http://sulcus. berkeley.edu
}

\begin{abstract}
An attractor modeling algorithm is introduced which draws upon techniques found in nonlinear dynamics and pattern recognition. The technique is motivated by the need for quantitative measures that are able to assess the stability of biological neural networks which utilize nonlinear dynamics to process information.
\end{abstract}

\section{Introduction}

In formulating neural networks which rely on nonlinear dynamics as an information processing medium [1] 2], two pertinent questions arise when the stability of the network is considered: 1) How stable is the network dynamics to input? 2 ) If one or more of the parameters in the network are altered, to what degree are the dynamics modified? These questions are central to understanding the utility of chaotic neural networks since their answers form the foundation for computational stability and robustness or, ultimately, the degree in which such systems can be relied on to perform extended parallel computations. However, of the present tools offered to dynamicists none are capable of answering these questions on a quantitative basis.

\subsection{Nonlinear System Identification}

To date a suite of nonlinear system identification methods have been introduced for dynamical systems, each geared toward solving a particular identification problem 3 3]. However, of the current methods offered in the literature, none are comparative in nature in the sense that approximative models are created for the sole purpose of comparison. In this paper a novel nonlinear system identification method is presented which relies on tools found in nonlinear dynamics and pattern recognition to form approximative models that lend themselves to comparison on a statistical basis. The density of states approach is founded upon the notion that a dynamical system has a corresponding set of attractors where the dynamical evolution of the system in a particular volume of phase space is governed by the attractor or its associated basin which occupies that space [5].

V.N. Alexandrov et al. (Eds.): ICCS 2001, LNCS 2074, pp. 231-234 2001.

(C) Springer-Verlag Berlin Heidelberg 2001 


\section{Attractor Density Models}

Using a set of trajectories originating from an independent and identically distributed initial condition set $\mathbf{S}$ intersecting a collection of attractors $\mathbf{A}$ or their associated basins $\mathbf{B}$, a particular region of state space can be independently explored. From a probabilistic reinterpretation of these independent state space trajectories a probability density model may be defined. Specifically, given a realization set $\{y\}$ generated from a known model $F(p)$ with unknown parameters or inputs $p$ acting on a known domain $\{x\}, F(p):\{x\} \rightarrow\{y\}$, a probability function may be estimated from a transformation of the set $\{y\}, P(T(\{y\}))$, such that $P(T(\{y\}))$ corresponds with $F(p):\{x\}$.

\subsection{Feature Selection and Segmentation}

The methodology used to form attractor density models is motivated by the two tenets of pattern recognition- Feature selection and segmentation of the feature set. Feature selection is carried out by decomposing independent explorations of state space, generated from a fixed domain, into reconstruction vectors. Segmentation of the feature vectors relies on exploiting the variance, covariance structure of a reconstruction vector set.

Given a time series $h\left(\mathbf{a}_{t}\right)=y_{t}$ where $A$ is a compact finite-dimensional set of states $\mathbf{a} \in A$ observed with a generic function $h, h: A \rightarrow \mathbf{R}$, the states a may be reproduced by $n$-dimensional vectors $\mathbf{b}$ produced by a linear operation on the time series values

$$
\mathbf{b}=M\left[h(\mathbf{a}), h\left(\mathbf{a}_{-\tau}\right), \ldots, h\left(\mathbf{a}_{-(n-1) \tau}\right)\right]^{T}
$$

where $\tau$ is a positive real number and $n \geq 2 d+1$ with box counting dimension $d$ [6]. In the present context of feature selection in a nonstationary process, the reconstruction operator $M=M_{4} M_{3} M_{2} M_{1}$ is comprised of three terms: $M_{1}$ finds and removes the mean from the realization, $M_{2}$ finds and normalizes the demeaned realization to unit energy, $M_{3}$ finds the spectral coefficients through the convolution of the remaining realization with a Morlet filter bank 7 . This has the effect of breaking each realization of the chaotic process into its dynamical modes. To segment each set of vectors, the dominant modes are extracted using singular value decomposition, $M_{4}$.

\subsection{Density Estimation}

Each dynamical state vector represents a state a of one of the attractors $A \in$ A. The density of trajectories in the neighborhood of state a can be viewed statistically in terms of having a certain probability of being visited. Using this notion an attractor representation may be built by calculating the density of states of an attractor in reconstruction space.

Under the assumption that each dynamical state vector is a sample from an unknown probability density function $f$, a kernel function $K$ provides a means 
of generating a nonparametric estimate $\hat{f}$ of $f$. The kernel density estimate $\hat{f}$ in dimension $d$ at point $\mathbf{a}^{\prime}$ with samples $\mathbf{a}_{i}$ is defined as [4]

$$
\hat{f}\left(\mathbf{a}^{\prime}\right)=\frac{1}{n h^{d}} \sum_{i=1}^{n} K\left(\frac{\left(\mathbf{a}^{\prime}-\mathbf{a}_{i}\right)^{T}\left(\mathbf{a}^{\prime}-\mathbf{a}_{i}\right)}{h^{2}}\right)
$$

where $K$ is defined as the multivariate Gaussian joint probability density function.

\section{A Test of Segmentation}

An interesting demonstration of this methodology is to see how well attractor density models are segmented for a range of parameter values in the MackeyGlass equation 8. First, attractor density models are built for each parameterization $(\tau=6,8, \ldots, 200, a=0.16,0.18, \ldots, 0.24)$ using forty realizations.
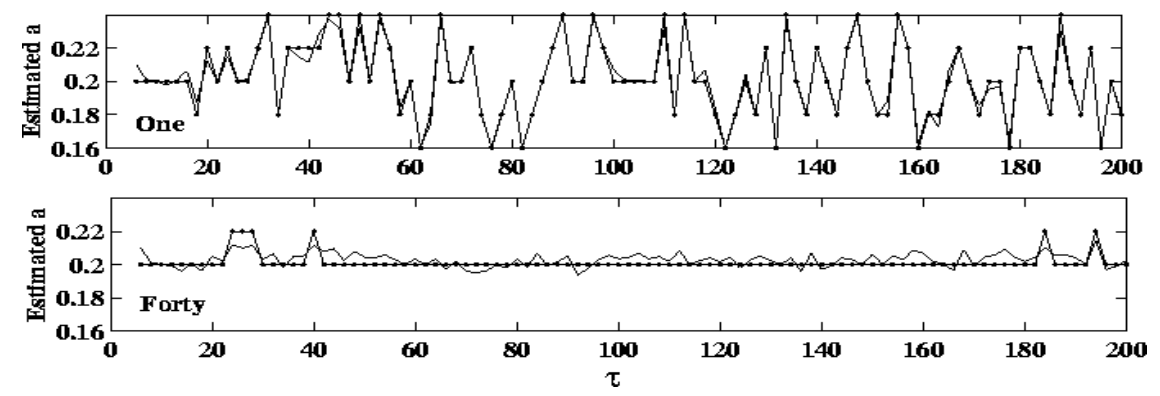

Fig. 1. Rounded estimates (dotted lines) of $a$ given one and forty realizations. The raw estimates are denoted by solid lines. The true value is $a=0.2$ for all values of $\tau$
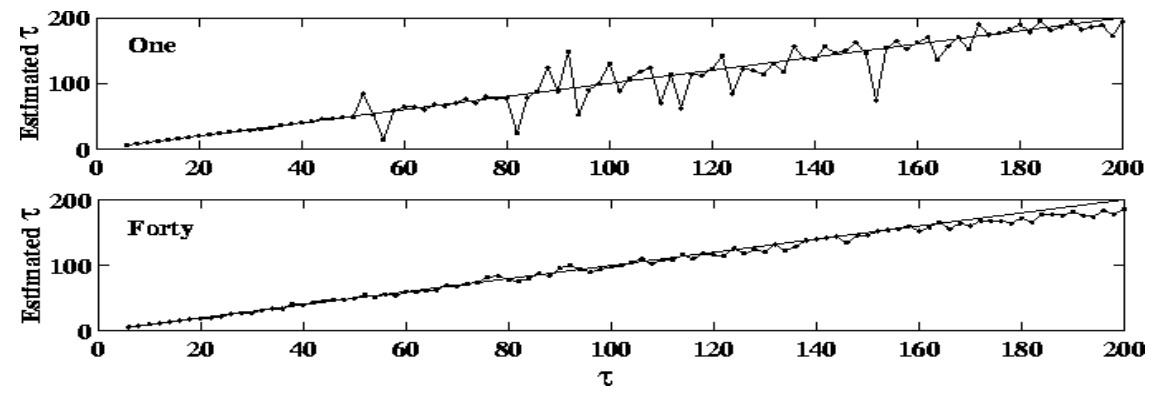

Fig. 2. Rounded estimates (dotted lines) of $\tau$ given one and forty realizations. The true values are denoted by the $45^{\circ}$ solid lines 
Given a set of one and forty realizations generated with unknown parameters $\tau=6,8, \ldots, 200$ and $a=0.20$, which model best describes the data? This question is answered by finding the center of mass of the density values found for each model using the points in each realization set. It is evident from the estimations of $a$ and $\tau$ in Figure 1 and 2, respectively, that increased sample sizes reduce the estimate variances indicating that with modest sample sizes a good degree of segmentation can be expected. The variability in the estimates is attributed to statistical fluctuations.

\section{Conclusion}

In this paper, the basis for creating attractor density models is described. The aim in formulating this technique lies in the need for nonlinear system identification methods which promote the comparison between models. With such methods, the ability to analyze the stability of chaotic neural networks to changes in internal parameters or input will be greatly improved.

This work was supported by ARO MURI grant ARO DAAH 04-96-0341.

\section{References}

1. Freeman, W.J.: Tutorial in Neurobiology: From Single Neurons to Brain Chaos. International Journal of Bifurcation and Chaos 2 (1992) 451-482

2. Kaneko, K., Tsuda, I.: Complex Systems: Chaos and Beyond. Springer-Verlag b, Berlin (2001)

3. Kosko, B.: Fuzzy Engineering. Prentice Hall, New Jersey (1997)

4. Silverman, B. W.: Density Estimation for Statistics and Data Analysis. Chapman and Hall, New York (1986)

5. Storm, C.: A Novel Dynamical Invariant Measure Addresses the Stability of the Chaotic KIII Neural Network. Proceedings of International Joint Conference on Neural Networks 1 (1999) 725-729

6. Sauer, T., Yorke, J. A., Casdagli, M.: Embedology. J. Stat. Phys. 65 (1991) 579-616

7. Teolis, A.: Computational Signal Processing with Wavelets. Birkhäuser, Boston (1998)

8. Mackey, M. C., Glass, L.: Oscillation and chaos in physiological control systems. Science 197 (1977) 287 